\title{
PHYSIOTHERAPY METHODS FOR PATIENTS WITH ADULT SCOLIOSIS
}

\author{
Evgeniya Dimitrova, Dimitar Nikolov \\ National Sports Academy „Vassil Levski“, Sofia, Bulgaria
}

\begin{abstract}
Spinal deformities are a major demographic health issue among the adult population all over the world. Surgeons are often very conservative in the treatment of adult scoliosis because of the complication rates associated with the surgeries. A prerequisite to surgical interventions is usually failure of all appropriate conservative care. There is currently a lack of consensus on the most efficient conservative treatments for adult scoliosis. The aim of the present study is to investigate the effect of two different methods of exercise-based therapies on the treatment of adult idiopathic scoliosis. The research was done among 62 patients with scoliosis $\left(10.93^{\circ} \mathrm{Cobb}\right)$ and back pain. They were randomly divided into two groups, depending on the physiotherapy (PT) protocol. To evaluate the effect of physiotherapy we used the posture assessment, visual analog scale (VAS) for pain, muscle strength test and Functional Rating Index (FRI). The control group - CG (n=37) performed a conventional physiotherapy program, including symmetrical exercises for the trunk muscles, lower and upper limbs, while the experimental group - EG (n=25) performed PT program, additionally including spinal mobilization, muscle energy technique, and postural therapy. The results after 6 months of treatment revealed a statistically signifcant improvement in the following parameters in the EG: the posture correction, pain index (according to VAS), muscle strength hand quality of live $(p<0.01)$. In the control group these results did not improve significantly. Conclusion: The combined use of spinal mobilization and postural therapy applied in the EG appears more effective than the conventional physiotherapy program used in the treatment of adult scoliosis.
\end{abstract}

Key words: adult scoliosis, idiopathic scoliosis, physiotherapy.

\section{INTRODUCTION}

The topic of the treatment of spine deformities in a skeletally mature individuals is interesting and up-to-date in modern society. Surgeons are often very conservative in the treatment of adult scoliosis because of the complication rates associated with the surgeries. A prerequisite to surgical interventions is usually failure of all appropriate conservative care. There is currently no consensus on the most effective non-surgical treatment of scoliosis after the age of 18 years. In a systematic literature study (Everett, Patel, 2007) of the non-surgical treatment of scoliosis after bone structure growth completion, the authors found only 10 articles in the Medline, PubMed and CINAHL associated with the use of kinesitherapy among these patients. The relationship between pain and scoliosis has been studied (Weiss et al., 1998). It is noted that among adults, scoliosis is associated with spinal pain - $60-80 \%$ of the patients (Daffner, Vaccaro, 2003), neurological symptoms (nerve root compression from the concave side with radial pain), sagittal and coronary imbalances, which also leads to back pain (Glassman et al., 2005). Attention is drawn to the need of study and improvement of the quality of life of women with idiopathic scoliosis (Freidel et al., 2002). A number of authors (Asher, Bur- 
ton, 2006; Hresko, 2013; Mayo et al., 1994; Negrini et al., 2006, 2012, 2015; Weinstein et al., 2003, 2008, 2013; Zadeh, Gleiber, 2015) recommend a report of the results for a clinically significant period and number of patients at the start and end of treatment with a scoliosis angle above $10^{\circ}$, above $30^{\circ}$, and over $50^{\circ}$ Cobb. According to them, epidemiology recognizes these degrees as risk limits for possible health consequences during increasing age, such as back pain and curvature progression. In the accessible literature there are no publications on the possibilities of kinesitherapy (KT) to reduce pain and improve the functional status of patients.

\section{AIM OF THE STUDY}

The aim of the present study is to investigate the effect of two different methods of exercise-based therapies on the treatment of adult idiopathic scoliosis.

\section{MATERIALS AND METHODS}

The research was done among 62 patients (aged between 23 and 44 years) with scoliosis $\left(10.93^{\circ} \mathrm{Cobb}\right)$ and back pain, after completion of the growth of bone structures (Risser 5). It was done during the period 2013-2016, in the Department of Physical Medicine and Rehabilitation at the Military Medical Academy, Sofia, Bulgaria. The group of patients was dominated by women - 41:21. The angle of curvature varied from $7^{\circ}$ to $18^{\circ}$ and averaged at $10.93^{\circ} \mathrm{Cobb}$. The division of patients into two groups - experimental group (EG) 37 patients with an average age of 31.18 years (range, 19-60, S.D. 8.07) and control group (CG) - 25 patients with an average age of 31.68 years (range, 18-59, S.D. 9.57) was done unintentionally and evenly. The collected data were statistically processed with SPSS software (Statistical Package for the Social Sciences), version 18.0, SPSS Inc. Chicago, IL, USA, and the following values were found: minimum, maximum, mean, S.D., and one sample paired t-test to compare pre- and posttreatment results of the group. The unpaired t-test was used to compare pre- and post-treatment results between the two groups, at a confidence level of $\mathrm{P} \leq 0.05$.

The comprehensive rehabilitation program, developed by a physician specialist in physical and rehabilitation medicine, included:

Preformed physical factors for pain reduction - magnetic therapy, phonophoresis, electrophoresis, interference currents, according to the estimation of the physician regarding the clinical condition of the patient.

\section{Kinesitherapy}

The aim of using kinesitherapy with both groups of patients was prevention of the scoliosis progress, back pain reduction and improvement of functional abilities.

In the control group (CG) a traditional physical therapy program was executed. It included: active axial elongation and mobilization of the spine, strengthening the trunk muscles, shoulder muscles, and hip joint muscles, coordination, balance and equilibrium of the body, correction of the posture, breathing and conditional exercises.

In the experimental group (EG), apart from the traditional physical therapy program, a specialized kinesitherapeutic method was also used:

Appropriate manual techniques and therapeutic exercises for mobilization and selfmobilization of the spine, neurodynamics techniques, muscle-energy techniques and massage were used for prevention and treatment of spinal pain syndrome.

The postural correction was achieved by training the patient in $3 \mathrm{D}$ self-correction of posture and postural control.

In order to stabilize the corrected posture and to prevent scoliosis progression, appropriate exercises were used for balance and strength of body muscles.

Maintenance and improvement of the 
spine mobility was achieved through manual joint mobilization techniques and exercises for range of movements.

The kinesitherapeutic means used with both groups of patients were dosed to medium and submaximal loads. Kinesitherapeutic behavior was consistent with the features of the pathological process, the degree of physical capacity, and the functional capacity of the neuromuscular apparatus. The selection of specific manual technique and its dosage was dependant on the clinical condition of the patient, the functional limitations and the physiological response of the patient, but in most of the cases it combined the different techniques and the dosage was gradually increased until the end of the immediate effect.

The following requirements were followed during the exercises:

Active axial elongation of the spine.

Unloading of the spine to create a normal position of the vertebrae and pain reduction.

Muscle tension in corrected position to stabilize the body.

Correction of posture and postural control in antigravity position.

Training patients for home-treatment which included holding the position of hypercorrection while lying flat for 15-20 minutes, once a day - up to 2-3 times a day, without provoking pain or discomfort.

The training was aimed at maintaining and improving spinal cord mobility while avoiding excessive stretching, rotation and lateral flexion.

The therapeutic effect was directly related to the correct selection and the skillful combination of the kinesitherapy means and followed the requirements of the specialized methodology. Great attention was paid to analytical symmetric and asymmetric isometric and isotonic strength exercises. The principle of an individual approach was strictly followed.

Lifting and carrying weights, long walks, jumps and spinal cord concussions, exercises and positions increasing the curvature or provoking pain were contra-indicated.

There were two courses of treatment in 10 procedures over six months. During the rest of the time, the patients were trained to perform a set of exercises to maintain the achieved results after the first cycle -30 minutes a day.

The following methods and tests were used to evaluate the functionalities of the patients before and after the rehabilitation course: history, examination, palpation, measurement of the trunk rotation by a scoliometer; visual analogue scale (VAS) for pain, measurement of the mobility of the spine and the length difference in the lower limbs in centimeters; static endurance of the trunk muscles; Functional Rating Index (FRI) (Bayar et al., 2004) and radiography - according to the opinion and prescription of an orthopedist.

\section{RESULTS AND ANALYSIS}

The data from the applied tests were assessed through statistical, comparative, variation and alternative analysis.

After an examination and analysis of the posture before and after the experiment, it became clear that there was a significant improvement in the posture among EG patients. These results, we think, were related to the use of 3D self-correction exercises with EG patients. They are based on pathokinesiological analysis and are actively reasoned, as well as correctly and systematically performed by patients. It was proven that they stimulate postural adjustment and reduction of asymmetry between the two body halves at the back, front and profile observations. For $\mathrm{CG}$, the percentage increment of posture improvement is minimal. Improvement of the posture among both groups of patients is in direct relation to the improvement of the dynamic functional indicators.

The follow-up of the trunk rotation at L4 level by scoliometry indicates that the im- 
provement among EG was $2.37^{\circ}$ while for the CG it was $1.4^{\circ}$. According to the SOSORT Scoliosis Guidelines (Negrini et al., 2012), a change of $5^{\circ}$ was considered clinically significant, but no significant changes in deformity were expected among patients after completion of spine growth.

The reported trend of improvements of all three levels of measurement were similar and confirms the effectiveness of the kinesitherapy, more expressed in EG, than in CG.

The variation analysis of VAS data for pain (Table 1) showed that the results in EG and CG were initially comparable. There was lower level of pain among CG patients, but the difference between the groups was statistically unreliable. At the end of the treatment, after two rehabilitation courses and a 6-month home rehabilitation, a statistically significant lower level of pain was reported among the patients from the EG compared to those from the $\mathrm{CG}(\alpha=0.05)$.

Table 1. Variation analysis of VAS data for pain.

\begin{tabular}{cccccccc}
\hline & EG & CG & & & & & \\
\hline & average X1 & average X2 & $\begin{array}{c}\mathbf{X}(\mathbf{X 1}-\Delta \\
\mathbf{( X 2}\end{array}$ & $\mathbf{S 1}$ & $\mathbf{S 2}$ & $\mathbf{t}$ & $\boldsymbol{\alpha}$ \\
\hline Beginning & 3,16 & 2,72 & 0,44 & 1,09 & 1,06 & 1,5 & - \\
\hline End & 0,41 & 0,84 & $-0,43$ & 0,49 & 0,74 & 2,55 & 0,05 \\
\hline $\mathrm{X} \Delta$ & 2,75 & 1,88 & & & & & \\
\hline $\mathrm{t}$ & 13,96 & 7,25 & & & & & \\
\hline$\alpha$ & 0,001 & 0,001 & & & & & \\
\hline
\end{tabular}

We associated the more expressed positive change among EG patients with the use of therapeutic massage, manual spinal mobilization techniques and self-mobilization exercises, as well as myorelaxation techniques and mobilization of the nervous system.

The measurements in centimeters at the end of the treatment indicated maintenance and slight improvement in spine mobility among both groups of patients. The possibility of active axial elongation of the spine in unloaded position, measured with the distance between $\mathrm{C} 7$ and $\mathrm{S} 1$, was minimal and varied between 0.6 and $1 \mathrm{~cm}$. Experience shows that patients perform better exercises of active axial elongation along the longitudinal axis of the spine in an antigravitational position. They find it very difficult to understand and coordinate their movements in an unloaded position. However, we executed these exercises in different body positions and combined them with every day activities, because, in our opinion, this is an important part of maintaining the spine in a good functional state and improving the nutrition of the intervertebral discs. We trained our patients in the morning before getting out of bed to always perform the active axial elongation exercise along the longitudinal axis of the spine as a prophylactic measure against degenerative disorders.

The statistical processing of the static endurance of the trunk muscles data shows a significant improvement among both groups of patients, when comparing beginning-end. Additionally, there was a proximity to the normal values $(\mathrm{EG} Z \mathrm{Zemp}=5.324$, CG Zemp $=3.755)$. Consequently, the applied kinesitherapy with both groups resulted in a significant improvement in the strength of the trunk muscles. The comparative analysis of the final results between the two groups showed statistically significant better outcomes among the EG pa- 
tients compared to CG patients ( $\alpha=0.05)$.

Before kinesitherapy, we found significant loss of functionality among all patients (Tables 2 and 3). The average value of the index was 20.94 points (EG) or FRI - 52.35\% loss of functionality and 20.24 points (CG) or FRI - $50.6 \%$ loss of functionality. The differences between the two groups were statistically unreliable and therefore the groups were comparable.

Table 2. Variation analysis of the results of Functional Rating Index (FRI).

\begin{tabular}{|c|c|c|c|c|c|c|c|}
\hline & EG & $\mathbf{C G}$ & & & & & \\
\hline & $\begin{array}{c}\text { FRI } \\
\text { average X1 }\end{array}$ & $\begin{array}{c}\text { FRI } \\
\text { average X2 }\end{array}$ & $\mathbf{X} \Delta$ & S1 & S2 & $\mathbf{t}$ & $\alpha$ \\
\hline Beginning & 20,94 & 20,24 & 0,7 & 2,19 & 2,13 & 1,27 & - \\
\hline End & 9,38 & 12,8 & $-3,42$ & 2,59 & 1,98 & 5,89 & 0,001 \\
\hline $\mathrm{X} \Delta$ & 11,58 & 7.44 & & & & & \\
\hline $\mathrm{t}$ & 20,78 & 12,8 & & & & & \\
\hline$\alpha$ & 0,001 & 0,001 & & & & & \\
\hline
\end{tabular}

The significant primary loss of functionality among both groups of patients was associated with mild to moderate pain, ranging from $25 \%$ to $75 \%$ of the day. With regard to daily living activities, the data varied from a lack of limitations to a need for everyday activity sassistance. More than $50 \%$ of the patients in both groups reported mild to moderate pain in long trips and part of the patients - in short trips. Spinal dysfunction had limited patients ${ }^{6}$ ability to work, with responses varying from „,can do usual work; no extra work" to "cannot work". We have found significant limitations as regards standing, walking, and weight lifting with all patients.

After the completion of the second rehabilitation course we recorded a statistically significant improvement $(\alpha=0.001)$ with both groups of patients (Table 2). Raising the level of functionality was directly related to improving the quality of life, which was an important task of kinesitherapy among these patients. In our opinion, there was undoubtedly a positive effect from the applied preformed physical factors, manual techniques and therapeutic exercises to reduce the pain syndrome. Pain reduction significantly improved the functional capabilities of the subjects under study (Table $3)$. At the end of the treatment, patients' ability to self-perform the everyday activities improved significantly; the percentage of patients reporting mild to moderate pain in long trips was reduced; patients' performance improved. Among $45 \%$ of the patients, there were some limitations as regards standing, walking, and lifting of heavy objects.

Table 3. Results of the FRI study (reported in percentages).

\begin{tabular}{rcc}
\hline & EG & CG \\
\hline & average X1 & average X2 \\
\hline Beginning & $52,35 \%$ & $50,60 \%$ \\
\hline End & $23,45 \%$ & $32,00 \%$ \\
\hline X $\Delta$ & $28,9 \%$ & $18,6 \%$ \\
\hline
\end{tabular}


We associate the positive functional results obtained from the two groups with the exercises executed for active elongation and mobilization of the spine, muscular strengthening and posture correction as part of the traditional kinesitherapy method. Besides, the results in EG of patients were associated with practicing additional kinesitherapy program exercises for active 3D spinal correction, manual mobilization and self-mobilization of the spine, neurodynamics techniques, exercises and manual techniques for improvement of the muscular balance.

On radiography we measured the Cobb's angle before the treatment's initiation and after the completion of the rehabilitation program (after 1 year). The results of the variance analysis showed that the scoliosis angle varies between $7^{\circ}$ and $16^{\circ}(\mathrm{Cobb})$ in the EG and between $8^{\circ}$ and $18^{\circ}$ in the CG. The variation coefficient was $6.1 \%$ for $\mathrm{EG}$ and $6.2 \%$ for $\mathrm{CG}$. Therefore, the sample was homogeneous. The average values were close for both groups. We did not notice any significant changes in Cobb angle's value after the applied treatment. The dynamics of the main radiological markers (Cobb angle) and the measurement data suggest that after the growth completion of the spine, a significant correction of the scoliosis was not possible.

\section{DISCUSSION}

The functional outcomes we gained after working with patients with scoliosis, after completion of the growth of the spine, confirm the results in research done by other authors. They claim that application of specific kinesitherapeutic exercises in case of scoliosis affects positively patients, in comparison with natural history of idiopathic scoliosis after 18 years of age (Negrini et al., 2015). The data obtained by us are comparable and better than those described by other authors (Morningstar, 2011, 2012). Scoliosis among adults is associated with a lower quality of life (Frei- del et al., 2002; Shapiro, Taira, Boachie-Adjei, 2003); low results from health questionnaire SF-36 (Schwab et al., 2003) and development of chronic pain (Weinstein et al., 2003). That is why, each treatment method that improves the quality of life, applied to our contingent of patients, expands and enriches the kinesitherapeutic practice. Additionally, it is advisable to explore the functional results in a sufficient contingent of patients for an extended period of time.

\section{CONCLUSIONS}

Kinesitherapy is an effective method for preventing the scoliosis'progression, reducing back pain and improving the functional capabilities of scoliosis patients after completion of bone structures growth.

The significantly higher positive growth in all of the main indicators in the experimental group compared with the control one indicates that the specialized methodology of kinesitherapy is more effective than the traditionally applied one.

The lack of consensual, effective, nonsurgical scoliosis treatment of these patients, imposes the necessity of the continuation and expansion of the research in this area, and the establishment of evidence-based clinical guidelines on kinesitherapy method, that effectively improves the functional capacities and patients' quality of life.

The results from our studies give us reason to recommend the inclusion of the specialized kinesitherapy methodology as part of the complex rehabilitation program for adult patients with scoliosis and back pain.

\section{REFERENCES}

Asher M, Burton D. (2006), Adolescent idiopathic scoliosis: natural history and long term treatment effects,Scoliosis, 1(1):2. doi:10.1186/1748-7161-1-2.

Bayar B, Bayar K, Yakut E, Yakut Y. (2004), Reliability and validity of the Func- 
tional Rating Index in older people with low back pain: preliminary report, Aging ClinExp Res, Vol. 16 No.1, pp. 49-52.

Daffner S, Vaccaro A. (2003), Adult degenerative lumbar scoliosis, Am $J$ Orthop (Belle Mead NJ). Vol. 32 No.2, pp. 77-82.

Everett CR, Patel RK. (2007), A systematic literature review of nonsurgical treatment in adult scoliosis, Spine, Vol. 32 No.9, pp. 130-4. doi: 10.1097/BRS.0b013e318134ea88.

Freidel K, Petermann F, Reichel D, Steiner A, Warschburger P, Weiss HR. (2002), Quality oflife in women with idiopathic scoliosis,Spine (Phila Pa 1976), Vol. 27 No.4, pp. 87-91.

Glassman SD, Bridwell K, Dimar JR, Horton W, Berven S, Schwab F. (2005), The impact of positive sagittal balance in adult spinal deformity, Spine (Phila Pa 1976), Vol. 30 No.18, pp. 2024-9.

Hresko MT. (2013), Clinical practice: idiopathic scoliosis in adolescents. $N$ Engl J Med,Vol. 368 No.9, pp. 834-41. doi: 10.1056/ NEJMcp1209063.

Mayo NE, Goldberg MS, Poitras B, Scott S, Hanley J. (1994), The Ste-Justine adolescent idiopathic scoliosis cohort study. Part III: Back pain,Spine (Phila Pa 1976), Vol. 19 No.14, pp. 1573-81.

Morningstar M. (2011), Outcomes For Adult Scoliosis Patients Receiving Chiropractic Rehabilitation: A 24-month Retrospective Analysis,J Chiropractic Medicine, Vol. 10 No.3, pp. 179-184.

Morningstar M. (2012), Scoliosis and ARC3D Therapy: Restoring Hope With NonSurgical Treatment,Infinity Publishing,pp. 8696.

Negrini A, Negrini MG, Donzelli S, Romano M, Zaina F, Negrini S. (2015), ScoliosisSpecific exercises can reduce the progression of severe curves in adult idiopathic scoliosis: a long-term cohort study, Scoliosis, 10:20. doi: 10.1186/s13013-015-0044-9.

Negrini S, Aulisa AG, Aulisa L, Circo AB, de Mauroy JC, Durmala J et al. (2012), 2011 SOSORT guidelines: orthopaedic and rehabilitation treatment of idiopathic scoliosis during growth, Scoliosis,7(1):3. doi: 10.1186/17487161-7-3.

Negrini S, Grivas TB, Kotwicki T, Maruyama T, Rigo M, Weiss HR. (2006), Members of the Scientific society On Scoliosis Orthopaedic and Rehabilitation Treatment (SOSORT): why do we treat adolescent idiopathic scoliosis? What we want to obtain and to avoid for our patients: SOSORT 2005 consensus paper, Scoliosis, 1:4. doi: 10.1186/17487161-1-4.

Schwab F, Dubey A, Pagala M, Gamez L, Farcy J. (2003), Adult scoliosis: a health assessment analysis by SF-36, Spine,Vol. 28 No.6, pp. 602-606.

Shapiro G, Taira G, Boachie-AdjeiO. (2003), Results of surgicaltreatment of adultidiopathicscoliosiswithlowbackpainandspinalstenosis: a study of long-termclinicalradiogr aphicoutcomes,Spine, Vol. 28 No.6, pp. 358363.

Weinstein SL, Dolan LA, Cheng JC, Danielsson A, Morcuende JA. (2008), Adolescent idiopathic scoliosis,Lancet, 371(9623):152737. doi: 10.1016/S0140-6736(08)60658-3.

Weinstein SL, Dolan LA, Spratt KF, Peterson KK, Spoonamore MJ, Ponseti IV. (2003), Health and function of patients with untreated idiopathic scoliosis: a 50-year natural history study, JAMA,289(5):559-67. doi:10.1001/ jama.289.5.559.

Weinstein SL, Dolan LA, Wright JG, Dobbs MB. (2013), Design of the Bracing in Adolescent Idiopathic Scoliosis Trial (BrAIST),Spine (Phila Pa 1976), Vol. 38 No.21, pp. 1832-41. doi: 10.1097/01.brs.0000435048.23726.3e.

Weiss HR, Verres C, Lohschmidt K, El 
Obeidi N. (1998), Pain and scoliosis - is there Idiopathic Scoliosis: An in Depth Analyany relationship?, Orthop Prax, Vol. 34, pp. sis and Historical Review, MOJ Orthop 602-606.

Rheumatol,Vol. 3 No.4, pp. 306-34.

Zadeh J, Gleiber M.(2015), Adolescent

Correspondence:

Evgeniya Dimitrova

National Sports Academy "Vassil Levski"

Department "Physiotherapy"

1 Gurgulyat Str., Sofia 1000

Bulgaria

E-mail: janydim@abv.bg 